\title{
Multi-Sensor Estimation Using Energy Harvesting and Energy Sharing
}

\author{
Steffi Knorn, Subhrakanti Dey, Anders Ahlén and Daniel E. Quevedo
}

\begin{abstract}
This paper investigates an optimal energy allocation problem for multi sensor estimation of a random source where sensors communicate their measurements to a remote fusion centre (FC) over orthogonal fading wireless channels using uncoded analog transmissions. The $\mathrm{FC}$ reconstructs the source using the best linear unbiased estimator (BLUE). The sensors have limited batteries but can harvest energy and also transfer energy to other sensors in the network. A distortion minimization problem over a finite-time horizon with causal and non-causal information is studied and the optimal energy allocation policy for transmission and sharing is derived. Several structural necessary conditions for optimality are presented for the two sensor problem with non-causal information and a horizon of two time steps. Numerical simulations are included to illustrate the theoretical results.
\end{abstract}

Index Terms - multi-sensor estimation, energy harvesting, energy sharing, optimal energy allocation

\section{INTRODUCTION}

Advances in the field of wireless communication have enriched many practical applications. A key role in this development is played by wireless sensors that measure a signal of interest and transmit the measurement to a remote estimator or Fusion Centre (FC). As wireless sensors have become not only more powerful but also more affordable and compact, they are used in many areas such as environmental data gathering [1], industrial process monitoring [2], mobile robots and autonomous vehicles [3], and monitoring of smart electricity grids [4].

Sensors are often located in remote places and therefore sometimes cannot be connected to reliable power sources. Even if connecting sensors to the electricity grid is feasible, it may be beneficial not to do so to simplify the installation process, facilitate changing the position of sensors or ensure sensors are independent of the power grid. Thus, sensors are often powered by batteries. Relying on battery power involves another significant restriction: As changing batteries is usually costly and undesirable, sensors have to be designed such that the limited available energy in the battery is used in the most efficient way, see [5]-[7] and the references therein.

One way to help overcome the limitation of finite battery capacity is to use energy harvesting. Often sensors are placed in an environment where energy can be harvested using solar panels, wind mills or other devices. The harvested energy can then be used for data transmission to the FC or be stored in the battery for future use. Because of the unreliable nature of most renewable energy sources, allocating the available energy in an optimal fashion to ensure the best possible performance of the network is a challenging task, [8]-[12]. Wireless compressive sensing for energy harvesting sensor nodes has also been studied in [13].

Recently, the field of wireless energy transfer has gained

Steffi Knorn, Subhrakanti Dey and Anders Ahlén are with the Department of Engineering Sciences, Uppsala University, Sweden; Email: \{steffi.knorn, subhra.dey, anders.ahlen\}@angstrom.uu.se

Daniel E. Quevedo is with the School of Electrical Engineering and Computer Science, The University of Newcastle, NSW 2308, Australia; Email: dquevedo@ieee.org new attention among researchers in the area of wireless sensor networks (WSN). This is motivated by wireless energy transfer becoming more efficient and less costly and thus having the potential to be used to recharge batteries of future wireless sensors. It was shown in [14] that energy can be transferred between two resonant objects of the same resonant frequency efficiently. Efficiencies of over $50 \%$ were achieved for distances up to 2 meters. See also [15]. An increasing number of companies is showing an interest in developing wireless energy transfer products [16], [17]. Their applications range from charging small devices such as cell phones in coffee shops [18] to charging electric vehicles [19]. Apparently a lot of the necessary technology is readily available and it is merely a question of time when the application of wireless energy transfer becomes feasible in a wider range of technical areas [20]. Other researchers have investigated how to optimally transmit energy and information through wireless communication channels [21]-[25].

Some researchers have started to investigate the potential benefits wireless energy transfer could bring to WSN. A wireless sensor network with a fixed base station and a wireless charging vehicle driving from sensor to sensor was considered in [26], [27]. An optimal traveling path for the vehicle and an algorithm to minimise the energy usage were derived. As background to our current work, in [28] an optimal power allocation policy is derived and multiple conditions for optimality are given for a two-hop relay channel with one-way energy transfer from the source to the relay. In the same paper, a Gaussian two-way channel with one-way energy transfer is investigated. It is shown that the optimal energy allocation policy is a directional two-way water filling algorithm. Similar results are derived for a multiple access channel with one-way energy transfer.

This paper investigates a wireless sensor network used to remotely estimate an independent and identically distributed (i.i.d.) bandlimited Gaussian process. Sensor measurements are sent via fading wireless channels to the FC, which uses the best linear unbiased estimator (BLUE), [29], to obtain an estimate of the physical process. In contrast to related works such as [30], we consider that all sensors in addition to harvesting energy from their environment, can also share energy between neighbouring nodes. A finitetime horizon sum distortion at the FC is minimized with respect to the energy allocation (for transmission as well as sharing) policy at the sensors, subject to energy harvesting constraints. Both causal (using dynamic programming) and non-causal information (using standard convex optimization techniques) are considered. Several insightful structural results are provided for a two-sensor two-step non-causal energy allocation problem.

\section{System Model}

Consider a system with $M$ sensors individually measuring a process of interest $\theta(k)$. All measurements are subject to additive noise. The remote sensors can transmit information to a fusion centre (FC). The latter estimates $\theta(k)$ given the 


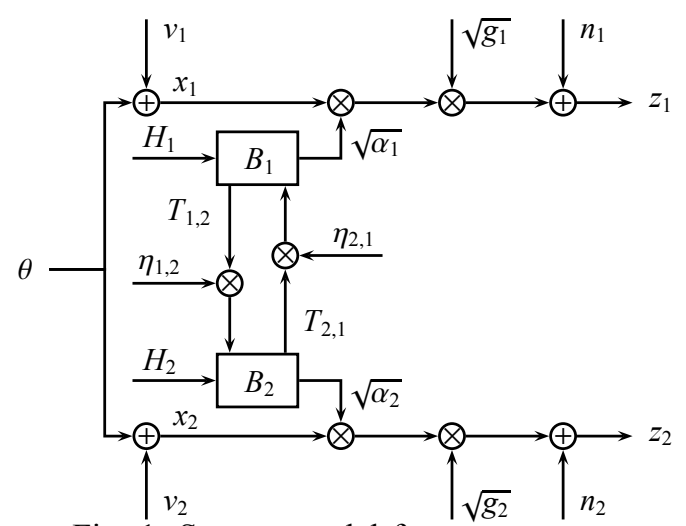

Fig. 1: System model for two sensors

available measurements. The transmitters adopt an analog amplify and forward uncoded strategy subject to additive noise. Every sensor node has an energy unit to harvest energy from its environment and a local battery whose energy can be used for data transmission. Additionally, each sensor has a unit to transmit and receive energy from other neighbouring sensors subject to transmission losses. A scheme showing a two sensor network is shown in Fig. 1.

\section{A. Source Model and Sensor Measurements}

It is assumed that the physical quantity of interest $\theta(k)$ is an i.i.d. Gaussian process with zero mean and variance $\sigma_{\theta}^{2}$. The measurements of sensor $m, x_{m}(k)$, is subject to measurement noise, $v_{m}(k)$, such that $x_{m}(k)=\theta(k)+v_{m}(k)$ for $1 \leq m \leq M$ and $k \geq 1$. The measurement noise processes $v_{m}(k)$ are assumed to be i.i.d. Gaussian, mutually independent of $\theta(k)$, having zero mean and variances $\sigma_{m}^{2}$.

\section{B. Transmission Model}

It is assumed that each sensor is attached to a transmitter and all transmitters adopt an uncoded analog amplify and forward strategy. ${ }^{1}$ This implies that at each time $k$, the transmitted signal from sensor $m$ is the measurement $x_{m}(k)$ amplified with the amplifying factor $\alpha_{m}(k)$. Assuming (without loss of generality) that each transmission interval is of duration unity, the necessary transmission energy with the amplifying factor $\alpha_{m}(k)$ is

$$
E_{m}(k)=\alpha_{m}(k)\left(\sigma_{\theta}^{2}+\sigma_{m}^{2}\right) .
$$

The channel power gain of the $m$ th channel (between sensor $m$ and the FC), $g_{m}(k)$, is assumed to be a non-negative i.i.d. process. Then, the received signal at the FC from sensor $m$ at time $k$ is given by $z_{m}(k)=\sqrt{\alpha_{m}(k) g_{m}(k)} x_{m}(k)+n_{m}(k)$ where the channel noise $n_{m}(k)$ is assumed to be i.i.d. additive white Gaussian noise with variance $\xi_{m}^{2}$.

\section{Energy Harvester, Energy Sharing and Battery Model}

Each sensor has access to an energy harvester that can gather energy from the environment. The amount of energy available to be harvested at sensor $m$ at time slot $k$, denoted by $H_{m}(k)$, is assumed unpredictable and described by an i.i.d. random process. Further, the harvested energy process and the channel gains are mutually independent. The energy harvested at time slot $k$ is stored in the battery and can be used for data transmission or energy sharing in the next time slot $k+1$. We denote that each sensor's maximal battery

\footnotetext{
${ }^{1}$ Analog transmission for multi-sensor estimation of a memoryless Gaussian source over a coherent multiaccess channel is optimal, [31] Further, the scheme does not need quantization or coding/decoding.
}

capacity is $\hat{B}_{m}$ and that transmitter $m$ consumes energy $E_{m}(k)$ from its battery to transmit data to the $\mathrm{FC}$ at $k$.

Each sensor is fitted with a unit to share energy with neighbouring nodes, that is, to transmit and receive energy to and from neighbouring nodes. Let this energy, transferred from sensor $m$ to $n$, be denoted $T_{m, n}$. The set of neighbouring nodes from which sensor $m$ can receive energy is denoted by $\mathcal{N}_{\mathrm{R}, m}$ and the set of neighbouring nodes to which sensor $m$ can transmit energy is denoted by $\mathcal{N}_{\mathrm{T}, m}$. Transferring energy is subject to losses. The efficiency of the energy transfer link from sensor $m$ to sensor $n$ is given by $\eta_{m, n}$ and is assumed to be static and strictly less then 1. Then the dynamics of the battery level of sensor $m$ is described by

$$
\begin{aligned}
B_{m}(k+1)= & \min \left\{B_{m}(k)+H_{m}(k)-E_{m}(k)-\sum_{n \in \mathcal{N}_{\mathrm{T}, m}} T_{m, n}(k)\right. \\
& \left.+\sum_{n \in \mathcal{N}_{\mathrm{R}, m}} \eta_{n, m} T_{n, m}(k), \quad \hat{B}_{m}\right\}
\end{aligned}
$$

\section{Distortion Measure at the Fusion Center}

At the $\mathrm{FC}$ given the vector of received signals $\mathbf{z}(k)=$ $\left(z_{1}(k), z_{2}(k), \ldots, z_{M}(k)\right)$, the BLUE [29], is used to calculate the estimate $\hat{\theta}(k)$, as per:

$$
\hat{\theta}(k)=\left(\mathbf{h}^{\mathrm{T}}(k) \mathbf{R}^{-1}(k) \mathbf{h}(k)\right)^{-1} \mathbf{h}^{\mathrm{T}}(k) \mathbf{R}^{-1}(k) \mathbf{z}(k)
$$

where $\mathbf{h}(k) \in \mathbb{R}^{M \times 1}$ with entries $h_{m}(k)=\sqrt{\alpha_{m}(k) g_{m}(k)}$, $\mathbf{R}(k) \in \mathbb{R}^{M \times M}$ is a diagonal matrix where $R_{m, m}(k)=$ $\sigma_{m}^{2} \alpha_{m}(k) g_{m}(k)+\xi_{m}^{2}$. Then, the variance of $\hat{\theta}(k)$, or distortion in reconstruction, is given by

$$
\operatorname{Var}(\hat{\theta}(k))=\left(\sum_{m=1}^{M} \frac{\alpha_{m}(k) g_{m}(k)}{\sigma_{m}^{2} \alpha_{m}(k) g_{m}(k)+\xi_{m}^{2}}\right)^{-1} .
$$

Denoting $d_{m}\left(E_{m}(k), s_{m}(k)\right)=\frac{E_{m}(k) s_{m}(k)}{1+\sigma_{m}^{2} E_{m}(k) s_{m}(k)}$ and $s_{m}(k)=$ $\frac{g_{m}(k)}{\xi_{m}^{2}\left(\sigma_{\theta}^{2}+\sigma_{m}^{2}\right)}$ with $E_{m}(k)$ in (1), the achieved distortion at time step $k$ is

$$
D(\mathbf{E}(k), \mathbf{s}(k))=\left\{\begin{array}{ll}
\frac{1}{\sum_{m} d_{m}\left(E_{m}(k), s_{m}(k)\right)} & \text { if } \sum_{m} E_{m}(k) g_{m}(k)>0 \\
\sigma_{\theta}^{2} & \text { if } \sum_{m} E_{m}(k) g_{m}(k)=0
\end{array} .\right.
$$

where $\mathbf{E}(k)=\left(E_{1}(k), E_{2}(k), \ldots, E_{M}(k)\right)$, and $\mathbf{s}(k)=$ $\left(s_{1}(k), s_{2}(k), \ldots, s_{M}(k)\right)$. In case no sensor is transmitting (or $g_{m}(k)=0$ for all $\left.m\right)$, the best estimate is $\hat{\theta}(k)=E(\theta(k))=0$ and the distortion $D(0, s(k))=\sigma_{\theta}^{2}$.

III. Finite-Time Horizon Optimal Transmission Energy and Energy Sharing Allocation Problem and its Solution

\section{A. Problem Formulation}

In this section it will be discussed how to choose the optimal transmission energy for information transmission to the FC and the optimal energy for sharing between sensors to minimise the expected finite-time horizon sum distortion at the FC under the assumption that complete causal information is available at the FC. Under this information pattern, all sensors report their current battery levels and their estimated channel gains (achieved via pilot transmissions from the FC and channel reciprocity) to the $\mathrm{FC}$ via a control channel during the beginning of a transmission slot. Thus, the information available at the $\mathrm{FC}$ at $k$ is $\mathcal{I}_{k}=$ $\left\{\mathbf{g}(k), \mathbf{B}(k), \mathcal{I}_{k-1}\right\}$ where $\mathbf{g}(k)=\left(g_{1}(k), g_{2}(k), \ldots, g_{M}(k)\right)$ and $\mathbf{B}(k)=\left(B_{1}(k), B_{2}(k), \ldots, B_{M}(k)\right)$ are the complete vectors of all channel gains and battery levels at time $k$, and $\mathcal{I}_{1}=\{\mathbf{g}(1), \mathbf{B}(1)\}$. 
An energy allocation policy is a set of functions to determine $\left\{\left(E_{m}(k),\left\{T_{m, n}(k)\right\}\right): m \in M\right.$, and $\left.n \in \mathcal{N}_{\mathrm{T}, m}\right\}$. It is feasible if the energy constraints $E_{m}(k) \geq 0, T_{m, n}(k) \geq 0$ and $E_{m}(k)+\sum_{n \in \mathcal{N}_{\mathrm{T}, m}} T_{m, n}(k) \leq B_{m}(k)$ are almost surely (a.s.) satisfied for all $1 \leq m, n \leq M$ and $k \geq 1$. The admissible control set is then the set of all possible energy allocation policies which are based only on the causal information set $\mathcal{I}_{k}$ and do not violate the energy constraints $E_{m}(k) \geq 0$, $T_{m, n}(k) \geq 0$ and $E_{m}(k)+\sum_{n \in \mathcal{N}_{T} m} T_{m, n}(k) \leq B_{m}(k) \forall m$. Define $\mathbf{T}(k)$ as the matrix with entries $(\mathbf{T}(k))_{m, n}=T_{m, n}(k)$ for $n \in$ $\mathcal{N}_{\mathrm{T}, m}$ and $(\mathbf{T}(k))_{m, n}=0$ otherwise. The finite-time horizon optimal transmission energy and energy sharing allocation problem which minimises the expected sum distortion over a finite horizon subject to energy harvesting constraints is given by

$$
\begin{aligned}
& \min _{\mathbf{E}(k), \mathbf{T}(k): 1 \leq k \leq K} \sum_{k=1}^{K} \mathbb{E}[D(\mathbf{E}(k), \mathbf{s}(k))] \\
& \text { s.t. } E_{m}(k), T_{m, n}(k) \geq 0 \text { and } E_{m}(k)+\sum_{n \in \mathcal{N}_{\mathrm{T}, m}} T_{m, n}(k) \leq B_{m}(k)
\end{aligned}
$$

a.s. for $1 \leq m, n \leq M$ and $1 \leq k \leq K$, and $B_{m}(k)$ satisfies (2).

\section{B. Finite-Time Horizon Optimal Energy Allocation Policy}

For the causal information case, the solution to the stochastic control problem (6) is given by Theorem 1:

Theorem 1: Consider $K \geq 1$ and let the initial condition be $I_{1}=\{g(1), B(1)\}$. Then the value of the finite-time horizon minimisation problem (6) with causal information is given by $V_{1}(\mathbf{g}(1), \mathbf{B}(1))$, which can be computed recursively from the backward Bellman dynamic programming equation

$$
\begin{aligned}
V_{k}(\mathbf{g}(k), \mathbf{B}(k))= & \min _{\mathbf{E}(k), \mathbf{T}(k)}\{D(\mathbf{E}(k), \mathbf{s}(k)) \\
& \left.+\mathbb{E}\left[V_{k+1}(\mathbf{g}(k+1), \mathbf{B}(k+1)) \mid \mathbf{E}(k), \mathbf{T}(k)\right]\right\}
\end{aligned}
$$

for $1 \leq k \leq K-1$ such that $E_{m}(k) \geq 0, T_{m, n}(k) \geq 0$ and $E_{m}(k)+\sum_{n \in \mathcal{N}_{\mathrm{T}, m}} T_{m, n}(k) \leq B_{m}(k)$ with the battery dynamic equation (2) for all $m$. In (7) the expectation is computed over the random channel gains and harvested energy levels and the terminal condition is

$$
V_{K}(\mathbf{g}(K), \mathbf{B}(K))=D(\mathbf{B}(K), \mathbf{s}(K))
$$

where all remaining energy is used for transmission in the final time $K$.

Proof: The proof follows from the optimality equations for finite-time horizon stochastic control problems. See for instance [32].

The solution to the allocation problem (6) is thus

$$
\begin{aligned}
\left\{\mathbf{E}^{o}(\mathbf{g}(k), \mathbf{B}(k)), \mathbf{T}^{o}(\mathbf{g}(k), \mathbf{B}(k))\right\}=\underset{\mathbf{E}(k), \mathbf{T}(k)}{\operatorname{argmin}}\{D(\mathbf{E}(k), \mathbf{s}(k)) \\
\left.+\mathbb{E}\left[V_{k+1}(\mathbf{g}(k+1), \mathbf{B}(k+1)) \mid \mathbf{E}(k), \mathbf{T}(k)\right]\right\}
\end{aligned}
$$

for $1 \leq k \leq K-1$ such that $E_{m}(k) \geq 0, T_{m, n}(k) \geq 0$ and $E_{m}(k)+\sum_{n \in \mathcal{N}_{\mathrm{T}, m}} T_{m, n}(k) \leq B_{m}(k)$ with the battery dynamics equation (2) for all $m$, and $V$ is the solution to the Bellman equation (7).

In general the solution to the dynamic programming equation (9) can only be obtained numerically, as there is no closed form solution. Since this numerical approach relies on computing the optimal policy for a large number of discretised channel gain and battery level values, we assume that this computation is done off-line at the FC (which has access to potentially unlimited energy and higher computational power) and stored in a look-up table. In realtime, as the FC receives the channel gains and battery level information of all sensors at the beginning of each transmission phase, it looks up the optimal energy allocation policies for the corresponding nearest discretised values of the channel gains and battery levels, and informs all the sensors via feedback, which is assumed to be delay-free and error-free. The sensors subsequently use these optimal energy allocation policies for data transmission and energy sharing. It should be noted that the communication overhead between the sensors and the FC for reporting channel gains and battery levels also consumes energy, which is not taken into account in this work. ${ }^{2}$

\section{Finite-Time Horizon Optimal Energy Allocation Problem Given Non-Causal Information and Unlimited BATTERY CAPACITY}

\section{A. Problem Formulation}

In this section we derive the optimal energy allocation policy for the MSE problem (6) given a finite-time horizon $K$ and a priori knowledge of the channel gains and the harvested energies for $k=1, \ldots K$ and all sensors. It will also be assumed that each sensor has an unlimited battery capacity, such that the battery level equation is simply

$$
\begin{aligned}
B_{m}(k+1)= & B_{m}(k)+H_{m}(k)-E_{m}(k) \\
& -\sum_{n \in \mathcal{N}_{\mathrm{T}, m}} T_{m, n}(k)+\sum_{n \in \mathcal{N}_{\mathrm{R}, m}} \eta_{n, m} T_{n, m}(k) .
\end{aligned}
$$

It is the aim to find the optimal energy allocation that solves the optimisation problem in (6) given complete non-causal information on all future channel gains and harvested energies. Since all information is available, a deterministic optimisation problem can be formulated and solved. Clearly, such a non-causal information pattern is not practical. However, the motivation for considering this is that the optimized distortion performance with the noncausal information serves as a lower bound and a benchmark for the optimized distortion performance with causal information derived in the previous section. The optimal energy allocation problem for the non-causal information pattern can be solved using standard convex optimization techniques as explained below. The convexity of the objective function has been shown in [33], [30], and also earlier in [34]. The energy harvesting constraints are linear in the transmission energy and shared energy variables, which renders the optimization problem convex.

\section{B. Lagrangian Formulation}

The Lagrangian formulation for this problem is given by

$$
\begin{aligned}
\mathcal{L}(\mathbf{E}, \mathbf{T}, \lambda)= & \sum_{k=1}^{K}[D(\mathbf{E}(k), \mathbf{s}(k)) \\
& +\sum_{m=1}^{M} \lambda_{m, k}\left(\sum_{l=1}^{k} E_{m}(l)-\sum_{l=1}^{k-1} H_{m}(l)-B_{m}(1)\right. \\
& \left.\left.+\sum_{l=1}^{k} \sum_{n \in \mathcal{N}_{T, m}} T_{m, n}(l)-\sum_{l=1}^{k-1} \sum_{n \in \mathcal{N}_{R, m}} \eta_{n, m} T_{n, m}(l)\right)\right] .
\end{aligned}
$$

${ }^{2}$ If this energy consumption is constant for each transmission slot, then it can be easily taken into account by subtracting this energy from the maximum battery level and defining a modified maximum battery level for each sensor. 
$E_{m}^{o}(k), T_{m, n}^{o}(k)$, and $\lambda_{m, k}$ are primal and dual optimal solutions to (11) if they satisfy the Karush-Kuhn-Tucker (KKT) necessary and sufficient optimality conditions for all $m, n$ and all $k$.

$$
\begin{aligned}
& E_{m}^{o}(k) \geq 0, \quad T_{m, n}^{o}(k) \geq 0, \quad \lambda_{m, k} \geq 0, \\
& \sum_{l=1}^{k} E_{m}^{o}(l)-\sum_{l=1}^{k-1} H_{m}(l)-B_{m}(1) \\
& +\sum_{l=1}^{k} \sum_{n \in \mathcal{N}_{T, m}} T_{m, n}(l)-\sum_{l=1}^{k-1} \sum_{n \in \mathcal{N}_{R, m}} \eta_{n, m} T_{n, m}(l) \leq 0, \\
& \lambda_{m, k}\left(\sum_{l=1}^{k} E_{m}(l)-\sum_{l=1}^{k-1} H_{m}(l)-B_{m}(1)\right. \\
& \left.+\sum_{l=1}^{k} \sum_{n \in \mathcal{N}_{T, m}} T_{m, n}(l)-\sum_{l=1}^{k-1} \sum_{n \in \mathcal{N}_{R, m}} \eta_{n, m} T_{n, m}(l)\right)=0, \\
& \left.\frac{\partial \mathcal{L}}{\partial E_{m}(k)}\right|_{E_{m}^{o}(k)} \begin{cases}\geq 0 & \text { for } E_{m}^{o}(k)=0 \\
=0 & \text { for } 0 \leq E_{m}^{o}(k) \\
& \leq B_{m}(k)-\sum_{n \in \mathcal{N}_{T, m}} T_{m, n}^{o}(k)\end{cases} \\
& \left.\frac{\partial \mathcal{L}}{\partial T_{m, n}(k)}\right|_{T_{m, n}^{o}(k)}\left\{\begin{array}{cc}
\geq 0 & \text { for } T_{m, n}^{o}(k)=0 \\
=0 & \text { for } 0 \leq \sum_{n \in \mathcal{N}_{T, m}} T_{m, n}^{o}(k) \\
& \leq B_{m}(k)-E_{m}^{o}(k) .
\end{array}\right.
\end{aligned}
$$

\section{Necessary Conditions for Energy Transfer}

In this subsection, necessary conditions for optimality of energy transfer between two sensors will be derived. The conditions depend on the inverted sum of future Lagrangian multipliers $v_{m, k}:=\left(\sum_{l=k}^{K} \lambda_{m, l}\right)^{-1}$.

As due to the KKT conditions $\lambda_{m, k} \geq 0$ for all $m$ and $k$, it follows that $v_{m, k} \leq v_{m, k+1}$ for all $m$ and $k<K$.

Lemma 1: If it is optimal to transmit energy from sensor $m$ to sensor $n$ at time $k$, that is $T_{m, n}^{o}(k)>0$, then $v_{n, k+1}=$ $\eta_{m, n} v_{m, k}$.

Proof: According to the KKT condition (16) it must be true that $\left.\frac{\partial \mathcal{L}}{\partial T_{m, n}(k)}\right|_{T_{m, n}^{o}(k)}=0$ for $T_{m, n}^{o}(k)>0$. Thus, evaluating the derivative of the Lagrangian (11) with respect to $T_{m, n}^{o}(k)$ yields

$$
\left.\frac{\partial \mathcal{L}}{\partial T_{m, n}(k)}\right|_{T_{m, n}^{o}(k)}=\sum_{l=k}^{K} \lambda_{m, l}-\eta_{m, n} \sum_{l=k+1}^{K} \lambda_{n, l}=0 .
$$

The result then follows from the fact that $v_{m, k}:=$ $\left(\sum_{l=k}^{K} \lambda_{m, l}\right)^{-1}$.

The intuitive observation that transferring energy between two sensors in both directions in the same time slot is not optimal, can be formally established as follows.

Corollary 1: It is not optimal to transmit energy between any pair of nodes in both directions in the same time step, that is $T_{m, n}^{o}(k), T_{n, m}^{o}(k)>0$ for all $m, n$ and $k$.

Proof: Following a similar argument as in the proof of Lemma 1, a necessary condition for $T_{n, m}^{o}(k)>0$ is

$$
\left.\frac{\partial \mathcal{L}}{\partial T_{n, m}(k)}\right|_{T_{n, m}^{o}(k)}=\sum_{l=k}^{K} \lambda_{n, l}-\eta_{n, m} \sum_{l=k+1}^{K} \lambda_{m, l}=0 .
$$

Combining (17), (18) yields

$$
\sum_{l=k}^{K} \lambda_{m, l}=\eta_{m, n} \eta_{n, m} \sum_{l=k}^{K} \lambda_{m, l}-\eta_{m, n} \eta_{n, m} \lambda_{m, k}-\eta_{m, n} \lambda_{n, k} .
$$

Thus, for $T_{m, n}^{o}(k)>0$ and $T_{n, m}^{o}(k)>0$ the efficiency factors have to satisfy

$$
\eta_{m, n} \eta_{n, m}=1+\frac{\eta_{m, n} \eta_{n, m} \lambda_{m, k}+\eta_{m, n} \lambda_{n, k}}{\sum_{l=k}^{K} \lambda_{m, l}} \geq 1
$$

This implies that at least one of $\eta_{m, n}$, or $\eta_{n, m}$ must be $\geq$ 1. Since the efficiency factors are always strictly less than 1 , this is not possible. Hence it is not optimal to transmit energy between two sensors in both directions at the same time.

\section{Optimal Energy Allocation Policy}

The optimal policy to determine how much energy should be used to transfer data to the FC at any time step is given by the following result.

Theorem 2: Consider (6) with the objective function replaced by $\sum_{k=1}^{K} D(\mathbf{E}(k), \mathbf{s}(k))$. Suppose that all sensors have unlimited battery capacities and access to non-causal information on the harvested energies and channel gains for all time steps and all sensors. Then the optimal energy allocation at time $k$ at sensor $m$ is given by

$$
E_{m}^{o}(k)= \begin{cases}0 & \text { if } D\left(\Omega^{o}(k), s(k)\right) \geq \sigma_{\theta}^{2} \\ \Omega_{m}^{o}(k) & \text { if } D\left(\Omega^{o}(k), s(k)\right)<\sigma_{\theta}^{2}\end{cases}
$$

where $\Omega^{o}(k)=\left(\Omega_{1}^{o}(k), \Omega_{2}^{o}(k), \ldots, \Omega_{M}^{o}(k)\right)^{\mathrm{T}}$ with

$$
\Omega_{m}^{o}(k)= \begin{cases}0 & \text { if } \Omega_{m}(k) \leq 0 \\ \Omega_{m}(k) & \text { if } 0<\Omega_{m}(k)<B_{m}^{*}(k) . \\ B_{m}^{*}(k) & \text { if } \Omega_{m}(k) \geq B_{m}^{*}(k)\end{cases}
$$

In (22),

$$
\Omega_{m}(k)=\frac{D_{k} \sqrt{v_{m, k}}}{\sigma_{m}^{2} \sqrt{s_{m}(k)}}-\frac{1}{\sigma_{m}^{2} s_{m}(k)}
$$

with the overall achieved distortion at time $k$ denoted by $D_{k}$ and the largest possible energy for data transmission

$$
\begin{aligned}
B_{m}^{*}(k)= & B_{m}(0)+\sum_{l=1}^{k-1} H_{m}(l)-\sum_{l=1}^{k-1} E_{m}^{o}(l) \\
& +\sum_{l=1}^{k-1} \sum_{n \in \mathcal{N}_{R, m}} \eta_{n, m} T_{n, m}^{o}(l)-\sum_{l=1}^{k-1} \sum_{n \in \mathcal{N}_{T, m}} T_{m, n}^{o}(l) .
\end{aligned}
$$

Proof: The KKT condition (15) for $E_{m}^{o}(k)>0$ yields

$$
\left.\frac{\partial \mathcal{L}}{\partial E_{m}(k)}\right|_{E_{m}^{o}(k)}=-\frac{D_{k}^{2} s_{m}(k)}{\left(1+\sigma_{m}^{2} E_{m}^{o}(k) s_{m}(k)\right)^{2}}+\sum_{l=k}^{K} \lambda_{m, l}=0 \text {. }
$$

Setting $v_{m, k}=\left(\sum_{l=k}^{K} \lambda_{m, l}\right)^{-1}$ leads to

$$
\Omega_{m}(k)=\frac{D_{k} \sqrt{v_{m, k}}}{\sigma_{m}^{2} \sqrt{s_{m}(k)}}-\frac{1}{\sigma_{m}^{2} s_{m}(k)} .
$$

Whenever $\Omega_{m}(k)$ is within the achievable boundaries of 0 and the battery level $B_{m}^{*}(k)$ we have $\Omega_{m}^{o}(k)=\Omega_{m}(k)$. Otherwise $\Omega_{m}(k)$ will be saturated below at 0 and above at $B_{m}^{*}(k)$ to ensure the KKT conditions are satisfied.

In case choosing the optimal energy allocation policy $\Omega_{m}^{o}(k)$ leads to an overall distortion that is greater or equal to $\sigma_{\theta}^{2}$, it is optimal not to transmit any data, and to save the available energy for future use.

Remark 1: Note that the optimal policy (26) can be seen as a generalisation of the well known water-filling algorithm. A distinguishing aspect is that water can only flow in one direction in time due to energy causality constraints. In this sense, this optimal energy allocation policy is similar to the directional water-filling algorithm presented in [9]. However, in contrast to the non-decreasing 
water levels in [9], the water levels for the optimal energy allocation policy change randomly over time as they depend on the overall distortion and the channel gains. The bottom levels also change randomly over time since they depend on the channel gains.

\section{Special Case of Two Sensor Horizon 2 Problem with Non-Causal Information AND UnLIMITED BatTery CAPACITY}

Although there is a closed form expression for the optimal energy allocation policy for the finite horizon case with non-causal information, it is difficult to gain much insight into the solution for any general $K$. This section presents some structural properties of the optimal solution to the energy allocation problem of a simplified problem with two sensors, finite-time horizon $K=2$, non-causal information and unlimited battery capacities. These properties provide important insights into the optimal energy allocation solutions. These insights can be used to implement simple suboptimal energy allocation policies for the causal case also. thus, one can avoid the prohibitive complexity of dynamic programming at the cost of loss of optimality. Such suboptimal policies are currently being investigated and will be presented in an extended version of this work.

\section{A. Problem Formulation}

In the specialised case of 2 sensors and sum distortion minimisation over 2 time-steps, the aim is to find the energy allocation policy, that consists of the data transmission energies $E_{1}(1), E_{1}(2), E_{2}(1)$ and $E_{2}(2)$, and the energy sharing quantities $T_{1,2}(1), T_{1,2}(2), T_{2,1}(1)$ and $T_{2,1}(2)$. Clearly, it is optimal to use all energy in the battery at $K=2$ for data transmission to the FC. It is also optimal not to transfer any energy between the sensors at $K=2$ as it could only be used in the third time slot. Hence, the notation can be simplified by dropping the time index for the battery levels, the harvested energies, the energies used for data transfer, and the amount of energy shared.

\section{B. Lagrangian Formulation}

Using the simplifications discussed above, and recalling the energy constraints in (6) yields the associated Lagrangian

$$
\begin{aligned}
& \mathcal{L}\left(E_{1}, E_{2}, T_{1,2}, T_{2,1}, \lambda_{1}, \lambda_{2}\right)=D_{1}+D_{2} \\
& \quad+\lambda_{1}\left(E_{1}+T_{1,2}-B_{1}\right)+\lambda_{2}\left(E_{2}+T_{2,1}-B_{2}\right)
\end{aligned}
$$

where $D_{1}$ and $D_{2}$ are the distortions at time 1 and 2 . $E_{1}^{o}, E_{2}^{o}, T_{1,2}^{o}, T_{2,1}^{o}$ and $\lambda_{1}^{o}, \lambda_{2}^{o}$ are primal and dual optimal solutions to (27) if and only if they satisfy KKT optimality conditions, similar to the KKT conditions in (12)-(16).

\section{Necessary Condition for Optimality}

To simplify the analysis, we will use the notation:

$$
\begin{gathered}
X_{1,1}:=\frac{D_{1}^{2} s_{1}(1)}{\left(1+\sigma_{1} E_{1}^{o} s_{1}(1)\right)^{2}}, \\
X_{1,2}:=\frac{D_{2}^{2} s_{1}(2)}{\left(1+\sigma_{1}\left(B_{1}+H_{1}-E_{1}^{o}-T_{1,2}^{o}+\eta_{2,1} T_{2,1}^{o}\right) s_{1}(2)\right)^{2}},
\end{gathered}
$$

$$
X_{2,2}:=\frac{D_{2}^{2} s_{2}(2)}{\left(1+\sigma_{2}\left(B_{2}+H_{2}-E_{2}^{o}-T_{2,1}^{o}+\eta_{1,2} T_{1,2}^{o}\right) s_{2}(2)\right)^{2}}
$$
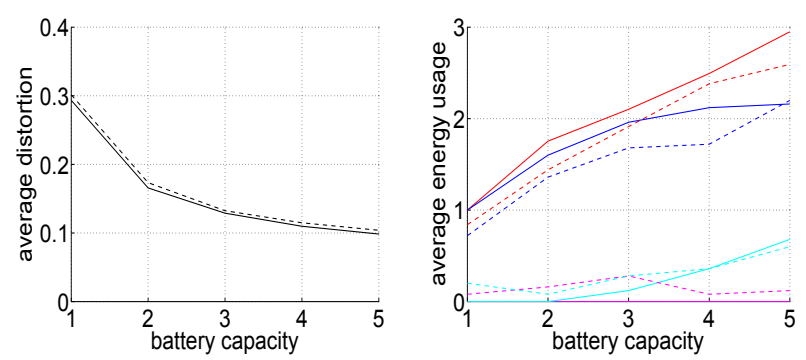

Fig. 2: Example 1: average distortion (black), $E_{1}^{o}$ (red), $E_{2}^{o}$ (blue), $T_{1,2}^{o}$ (magenta) and $T_{2,1}^{o}$ (light blue); for the noncausal case (solid lines) and the causal case (dashed lines)

The following result follows directly by examining the partial derivatives of the Lagrangian $\left.\frac{\partial \mathcal{L}}{\partial T_{1,2}}\right|_{T_{1,2}^{o}}$ and $\left.\frac{\partial \mathcal{L}}{\partial T_{2,1}}\right|_{T_{2,1}^{o}}$, and the proof is omitted.

Lemma 2: If it is optimal to transfer energy from sensor 1 to 2 at time 1 , that is $T_{1,2}^{o}>0$, then $X_{1,2} \leq \eta_{1,2} X_{2,2}$. Similarly, if it is optimal to transfer energy from sensor 2 to sensor 1, then one must have $X_{2,2} \leq \eta_{2,1} X_{1,2}$.

A necessary optimality condition for data transmission to the FC can be obtained in a similar manner:

Lemma 3: If it is optimal to transmit $x_{1}(1)$ to the $\mathrm{FC}$ at time 1 , that is $E_{1}^{o}>0$, then $X_{1,2} \leq X_{1,1}$.

Proof: Evaluating the derivative $\left.\frac{\partial \mathcal{L}}{\partial E_{1}}\right|_{E_{1}^{o}}$ together with the suitable KKT condition yields the result.

Finally, a necessary optimality condition for energy storing is simply $\lambda_{1}=0$.

\section{Examples and Numerical Results}

Example 1: A system with two sensors and a finite horizon of $K=5$ is simulated where $\eta_{1,2}=0.8, \eta_{2,1}=0.8$, where $g_{1}, g_{2}$ are each exponentially distributed with mean 4 , and $H_{1}$ and $H_{2}$ are chosen randomly using an exponential distribution with mean $4 m W$ each. Fig. 2 illustrates results for different battery capacities between $1 \mathrm{~mW}$ and $5 \mathrm{~mW}$ for the algorithm using non-causal information (solid lines) and causal information (dashed lines).

Increasing the battery capacities leads to an overall lower distortion. As expected, the average distortion is best for the algorithm using non-causal information, while the optimal algorithm (using full, causal information) performs almost as well as the algorithm with non-causal information.

The average energy used to transmit data to the FC from sensor 1 and 2 both increase with the battery capacity. While for this particular choice of random values, it is optimal not to transfer any energy from sensor 1 to 2 (with non-causal information), the optimal amount of energy to transfer from sensor 2 to 1 even increases in the non-causal case for an increase in the battery capacity. Hence, allowing energy transfer leads to better performance.

Example 2: A similar system as in the previous example with a finite horizon of $K=5$ is simulated. Here $g_{1}, g_{2}$ are exponentially distributed with mean 4 and 1 respectively, whereas $H_{1}$ and $H_{2}$ are chosen randomly using an exponential distribution with means $1 \mathrm{~mW}$ and $4 \mathrm{~mW}$ respectively. Hence, one sensor harvests on average more energy but has on average a worse channel compared to the second sensor. The battery capacities are fixed at $3 \mathrm{~mW}$ and the energy transfer efficiency varies between 0.2 and 0.8 .

The average distortion and the average energy usages are illustrated in Figure 3. The average distortion decreases in both cases (non-causal (solid line) and causal (dashed 

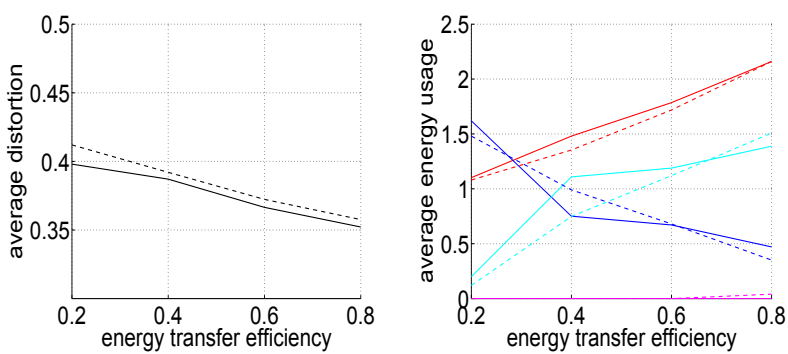

Fig. 3: Example 2: average distortion (black), $E_{1}^{o}$ (red), $E_{2}^{o}$ (blue), $T_{1,2}^{o}$ (magenta) and $T_{2,1}^{o}$ (light blue); for the noncausal case (solid lines) and the causal case (dashed lines)

line)) when the efficiency increases. This indicates that an increased energy transfer efficiency leads to performance improvements.

\section{Conclusions}

This paper studied optimal energy allocation in a sensor network where all sensors take noisy measurements of a random process and amplify and forward them via uncoded analog transmissions to a FC that reconstructs an estimate using BLUE. The sensors are capable of energy harvesting as well as energy sharing with neighbouring sensors. The optimal energy allocation policy for minimising the expected sum distortion for the finite-horizon case is studied with causal information and an arbitrary but finite maximum battery capacity, via dynamic programming methods. For the non-causal case with unlimited battery capacity, the optimal energy allocation policy is derived via convex optimisation techniques. In addition, some necessary optimality conditions for energy transfer between neighbouring sensors were given. Several explicit necessary optimality conditions are derived for the special case of two sensors with a finite-time horizon of 2 and unlimited battery capacities. These conditions provide insights into the trade-off amongst the sensors' decision to use energy for transmission, share energy with the other sensor or store energy for future use.

Numerical simulations showed that, upon increasing the battery capacity, the average distortion decreases both in the non-causal and the causal case. When increasing the energy transfer efficiency, the average distortion also decreases in case the sensors are asymmetric with regard to their average channel gains and average energy harvesting patterns.

Future research will study suboptimal decentralised policies that rely on local causal information at each sensor only, and require a substantially less computational effort than solving the Bellman dynamic programming equation, while still providing a reasonable distortion performance.

\section{REFERENCES}

[1] I. Akyildiz, W. Su, Y. Sankarasubramaniam, and E. Cayirci, "A survey on sensor networks," IEEE Communications Magazine, vol. 40, no. 8, pp. 102-114, August 2002.

[2] V. Gungor and G. Hancke, "Industrial wireless sensor networks: Challengesm design, principles and technical approaches," IEEE Transactions on Industrial Electronics, vol. 56, no. 10, pp. 42584265, 2009

[3] C.-Y. Chong and S. Kumar, "Sensor networks: Evolution, opportunities and challenges," Proceedings of the IEEE, vol. 91, no. 8, pp. 1247-1256, April 2003.

[4] V. Gungor, B. Lu, and G. Hancke, "Opportunities and challenges of wireless sensor networks in smart grid," IEEE Transactions on Industrial Electronics, vol. 57, no. 10, pp. 3557-3564, October 2010.

[5] C. E. Jones, K. M. Sivalingam, P. Agrawal, and J. C. Chen, "A survey of energy efficient network protocols for wireless networks," Wireless Networks, vol. 7, no. 4, pp. 343-358, 2001
[6] A. J. Goldsmith and S. B. Wicker, "Design challenges for energyconstrained ad hoc wireless networks," IEEE Wireless Communications, vol. 9, no. 4, pp. 8-27, 2002.

[7] D. E. Quevedo, A. Ahlén, and J. Østergaard, "Energy efficient state estimation with wireless sensors through the use of predictive power control and coding," IEEE Transactions on Signal Processing, vol. 58, no. 9, pp. 4811-4823, September 2010

[8] V. Sharma, U. Mukherji, V. Joseph, and S. Gupta, "Optimal energy managment policies for energy harvesting sensor nodes," IEEE Transactions on Wireless Communications, vol. 9, no. 4, 2010.

[9] O. Ozel, K. Tutuncuoglu, J. Yang, S. Ulukus, and A. Yener, "Transmission with energy harvesting nodes in fading wireless channels: Optimal policies," IEEE Journal of Selected Areas in Commnunication, vol. 29, no. 8, pp. 1732-1743, September 2011.

[10] J. Yang, O. Ozel, and S. Ulukus, "Broadcasting with an energy harvesting rechargeable transmitter," IEEE Transactions on Wireless Communications, vol. 11, no. 2, pp. 571-583, February 2012.

[11] C. K. Ho and R. Zhang, "Optimal energy allocation for wireless communications with energy harvesting constraints," IEEE Transactions on Signal Processing, vol. 60, no. 9, pp. 4808-4818, September 2012.

[12] A. Nayyar, T. Başar, D. Teneketzis, and V. Veervalli, "Optimal strategies for communication and remote estimation with an energy harvesting sensor," IEEE Transactions on Automatic Control, vol. 58, no. 9, pp. 2246-2260, September 2013.

[13] G. Yang, V. Tan, C. K. Ho, S. H. Ting, and Y. L. Guan, "Wireless compressive sensing for energy harvesting sensor nodes," IEEE Trans. Signal Process., vol. 61, no. 18, pp. 4491-4505, 2013.

[14] A. Kurs, A. Karalis, R. Moffatt, J. D. Joannopoulos, P. Fisher, and M. Soljačić, "Wireless power transfer via strongly coupled magnetic resonances," Science, vol. 317, no. 83, pp. 83-86, July 2007.

[15] A. Karalis, J. D. Joannopoulos, and M. Soljačić, "Efficient wireless non-radiative mid-range energy transfer," Annals of Physics, vol. 323, no. 1, pp. 34-48, January 2008.

[16] [Online]. Available: http://www.wirelesspowerconsortium.com

[17] [Online]. Available: http://www.powercastco.com

[18] [Online]. Available: http://money.cnn.com/2014/06/12/technology/... mobile/starbucks-recharging/

[19] [Online]. Available: http://www.pluglesspower.com

[20] W. Lumpkins, "Nikola tesla's dream realized: Wireless power energy harvesting," IEEE Consumer Electronics Magazine, vol. 3, no. 1, pp. 39-42, January 2014.

[21] A. M. Fouladgar and O. Simeone, "On the transfer of information and energy in multi-user systems," IEEE Communications Letters, vol. 16, no. 11, pp. 1733-1736, November 2012.

[22] R. Zhang and C. K. Ho, "MIMO broadcasting for simultaneous wireless information and power transfer," IEEE Transactions on Wireless Communications, vol. 12, no. 5, pp. 1989-2001, May 2013.

[23] L. Liu, R. Zhang, and K.-C. Chua, "Wireless information and power transfer: A dynamic power splitting approach," IEEE Transactions on Communications, vol. 61, no. 9, pp. 3990-4001, September 2013.

[24] K. Huang and E. G. Larsson, "Simultaneous information and power transfer for broadband wireless systems," IEEE Transactions on Signal Processing, vol. 61, no. 23, pp. 5972-5986, December 2013.

[25] K. Huang and V. K. N. Lau, "Enabling wireless power transfer in cellular networks: Architecture, modeling and deployment," IEEE Transactions on Wireless Communications, vol. 13, no. 2, 2014.

[26] Y. Shi, L. Xie, Y. T. Hou, and H. D. Sherali, "On renewable sensor networks with wireless energy transfer," in Proceedings IEEE INFOCOM, April 2011, pp. 1350-1358.

[27] L. Xie, Y. Shi, Y. T. Hou, W. Lou, H. D. Sherali, and S. F. Midkiff, "Renewable sensor networks with wireless energy transfer: The multi-node case," in 9th Annual IEEE Communications Society Conference on Sensor, Mesh and Ad Hoc Communications and Networks, 2012.

[28] B. Gurakan, O. Ozel, J. Yang, and S. Ulukus, "Energy cooperation in energy harvesting communications," IEEE Transactions on Communications, vol. 61, no. 12, pp. 4884-4898, December 2013.

[29] J. Mendel, Lessons in Estimation Theory for Signal Processing, ser. Communications and Control. Prentice Hall, 1995.

[30] M. Nourian, S. Dey, and A. Ahlén, "Distortion minimization in multisensor estimation with energy harvesting," accepted for publication in IEEE Journal on Selected Areas in Ccommunications, 2015.

[31] M. Gastpar, "Uncoded transmission is exactly optimal for a simple gaussian "sensor" network," IEEE Transactions on Information Theory, vol. 54, no. 11, pp. 5247-5251, November 2008.

[32] D. P. Bertsekas, Dynamic Programming and Optimal Control, 3rd ed. Athena Scientific, 1995, vol. 1.

[33] A. Limmanee, S. Dey, and A. Ahlén, "Distortion minimization via multiple sensors with energy harvesting," in Proceedings of the 14th IEEE SPAWC, June 2013, pp. 225-229.

[34] S. Cui, J. Xiao, A. J. Goldsmith, Z. Luo, and H. V. Poor, "Estimation diversity and energy effiiency in distributed sensing," IEEE Trans. Signal Process., vol. 55, no. 9, pp. 4683-4695, Sep. 2007. 